(C) 2016 IEEE. Personal use of this material is permitted. Permission from IEEE must be obtained for all other uses, in any current or future media, including reprinting/republishing this material for advertising or promotional purposes, creating new collective works, for resale or redistribution to servers or lists, or reuse of any copyrighted component of this work in other works. 


\title{
Aperture Efficiency Improvement Using Metasurface
}

\author{
H.L. Zhu' ${ }^{1}$, S.W. Cheung ${ }^{1}$, Y. Jay Guo ${ }^{2}$, Can Ding ${ }^{2}$, T.I. Yuk ${ }^{1}$ \\ ${ }^{1}$ Department of Electrical and Electronic Engineering, The University of Hong Kong, Hong Kong, zhuhl@eee.hku.hk \\ ${ }^{2}$ Globle Big Data Technologies Center, University of Technology Sydney, Can.Ding@uts.edu.au
}

\begin{abstract}
A method to improve the aperture efficiency of antennas using a non-uniform metasurface (MS) is studied in this paper. For illustration, a patch antenna and a planar MS having unit cells of square patches with different sizes are used. The sizes of the square patches on the MS follow a radial gradient index function. The aperture efficiencies of the patch antenna and the antenna using the MS are compared. For easy comparison, the patch antenna is designed to have an aperture size of $\lambda_{0} \times \lambda_{0}$, where the $\lambda_{0}$ is wavelength at the operating frequency in free space. Simulation results show that, by placing the MS atop the patch antenna with a separating distance of 35 $\mathrm{mm}$, the directivity of the patch antenna can be increased from $9.7 \mathrm{dBi}$ to $11.6 \mathrm{dBi}$. With the aperture size remains unchanged, the aperture efficiency of the patch antenna is increased by more than $100 \%$.
\end{abstract}

Index Terms-patch antenna, aperture efficiency, metasurface, radial gradient index function

\section{INTRODUCTION}

Owing to their compact planar structures, printed antennas are often used in portable wireless devices. Patch antennas, as a typical type of printed antennas having the advantages of low profile and high directivity, serve as good candidates for wireless communications terminals. However, the physical aperture size of a patch antenna on a wireless device is always limited.

When an electromagnetic wave impinges on an antenna, the power capturing characteristics of the antenna is related to its effective aperture area which is different from the physical aperture area of the antenna [1]. The maximum effective aperture $\left(\mathrm{A}_{\mathrm{em}}\right)$ of an antenna is related to its maximum directivity $\left(D_{0}\right)$ by $A_{e m}=\lambda^{2} /(4 \pi)^{*} D_{0}[1]$ and the aperture efficiency can be written as $E_{a e}=A_{e m} / A_{p h}$, where the $A_{p h}$ is the physical aperture size of the antenna [1]. Thus, the aperture efficiency of an antenna can be improved by increasing the directivity of the antenna, yet keeping the physical aperture size unchanged. For horn or reflector antennas in general, the aperture efficiency is from about $50 \%$ to $80 \%$ [2]. For the patch antenna studied in this paper, the aperture efficiency is around $78 \%$.

One popular method to increase the directivity is to place a partially reflective surface (PRS) or a high permittivity dielectric slab atop of the radiating aperture. However, the area of the PRS employed is usually much larger than that of the radiating aperture. It can lead to lower aperture efficiency than that of the antenna without PRS. Employing dielectric slab is a promising method to enhance the directivity without increasing the aperture size. But they are high cost and bulky.

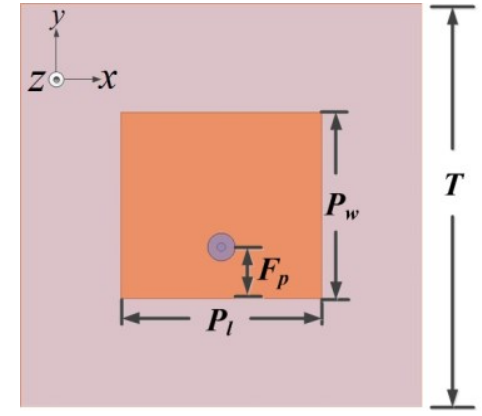

(a)

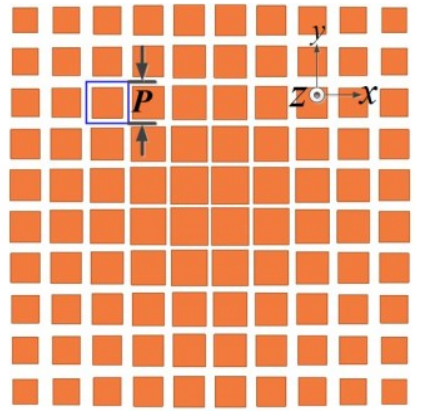

(b)
Fig. 1. Geometries of patch antenna and metasurface.

TABLE I. DIMENSIONS OF ANTENNA AND MS (UNIT:MM)

\begin{tabular}{|c|c|c|c|c|c|c|}
\hline $\boldsymbol{P}_{l}$ & $\boldsymbol{P}_{w}$ & $\boldsymbol{F}_{p}$ & $\boldsymbol{P}$ & $\boldsymbol{P}_{\max }$ & $\boldsymbol{T}$ & $\boldsymbol{H}$ \\
\hline 29 & 27 & 6.2 & 6 & 5.5 & 60 & 35 \\
\hline
\end{tabular}

Metasurface (MS), a two-dimensional equivalent of metamaterial, is essentially a surface distribution of electrically small scatterers [3]. MS is also able to enhance the gain when placed atop of a radiator. Compared to PRS, it has a much smaller size. In this paper, a non-uniform MS is proposed to increase the aperture efficiency of a patch antenna. Both the patch antenna and the MS are designed using planar technology to operating at $5 \mathrm{GHz}$. The MS is placed at a distance away and atop of the patch antenna. The EM simulation tool CST is used to carry out the study. The aperture efficiencies of the patch antenna alone and with the MS together are compared. For easy comparison, the area of the patch antenna has an area of $\lambda_{0} \times \lambda_{0}$, where the $\lambda_{0}$ is wavelength in free space, and the dielectric constant of the substrate is assumed to be the same as that of air. This is because if the relative dielectric constant of the substrate for antenna and MS are greater than 1, the effective aperture size of the antenna has to be rescaled and the recalculated results depend on not only the value of the dielectric constant but also the thickness of the substrate. To the best of the author's knowledge, by using the proposed single layer of MS with such a small area, the highest aperture efficiency is achieved compared to its counterparts [11-17]. 


\section{Design of Antenna AND Metasurface (MS)}

The patch antenna used in the study is shown in Fig. 1(a) and designed to operate at $5 \mathrm{GHz}$. A coaxial cable is used to directly feed the patch antenna through the ground plane and the substrate. The patch antenna together with the coaxial cable is designed on a double-sided substrate with a dielectric constant of 1 and a thickness of $1.6 \mathrm{~mm}$. The EM simulation tool CST is used to optimize the dimensions, in terms of directivity at the boresight direction and $\mathrm{S} 11$ at $5 \mathrm{GHz}$, with results listed in Table I.

The metasurface (MS) used is composed a number of unit cells in the forms of square patches with variable sizes, as shown in Fig. 2(b). The unit cells are placed periodically in the $x$ - and $y$ - directions of the substrate. To achieve higher directivity, the sizes of the square patches are varied according to a gradient index function, thus making the MS a gradient index (GRIN) material $[4,5]$. However, the size of lattice for each unit cell is fixed at $P$, as shown in Fig. 1(b). The largest four unit cells with a patch size of $\left(P_{\max }\right)^{2}=(5.5)^{2} \mathrm{~mm}^{2}$ are placed closest to the center of the MS. The unit cells further away from the center position of the MS have smaller sizes. The MS is placed atop of the patch antenna with a separating distance of $H=35 \mathrm{~mm}$ to form a metasurfaced antenna (MS antenna).

\section{RESULTS AND DISCUSSIONS}

The simulated reflection coefficients S11 of the patch antenna alone and the MS antenna are shown in Fig. 2. It can be seen that both the patch antenna and MS antenna have the same resonant frequency of $5 \mathrm{GHz}$. Thus the MS does not have much effect on the resonance of the patch antenna. The impedance bandwidths (for S11 $<-15 \mathrm{~dB}$ ) of the patch antenna and MS antenna are 4.9-5.08 and 4.9-5.1 GHz, respectively, so the MS only slightly change the bandwidth of the patch antenna.

The simulated directivities at the boresight direction of the patch antenna and MS antenna are shown in Fig. 3(a), while the simulated aperture efficiencies are shown in Fig. 3(b). It can be seen that, the patch antenna and the MS antenna have the directivities of $9.7 \mathrm{dBi}$ and $11.6 \mathrm{dBi}$ at $5 \mathrm{GHz}$, thus the MS increases the directivity by $1.9 \mathrm{~dB}$. As increasing directivity leads to increasing aperture efficiency as described previously. Fig. 3(b) shows that the aperture efficiency of the MS antenna reaches up to $117 \%$ at $5 \mathrm{GHz}$, which is due to the use of the MS. At $4.7 \mathrm{GHz}$, the aperture efficiency even reaches $121 \%$. However, the MS antenna cannot work at $4.7 \mathrm{GHz}$ because the $\mathrm{S} 11$ is only $-2 \mathrm{~dB}$ as shown in Fig. 2. These results show that, by using a MS, the effective aperture size of a patch antenna can be larger than its physical aperture size.

The simulated 3D radiation pattern of the patch antenna and the MS antenna are shown in Fig. 4(a) and (b), respectively. It can be seen that the radiation pattern of the MS antenna is more directional in boresight direction than that of the patch antenna.

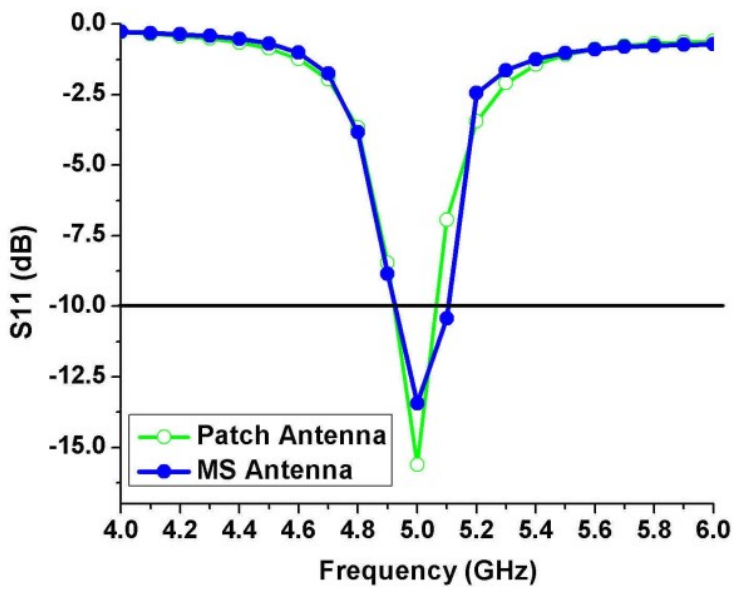

Fig. 2. Simulated S11 for patch antenna and MS antrenna

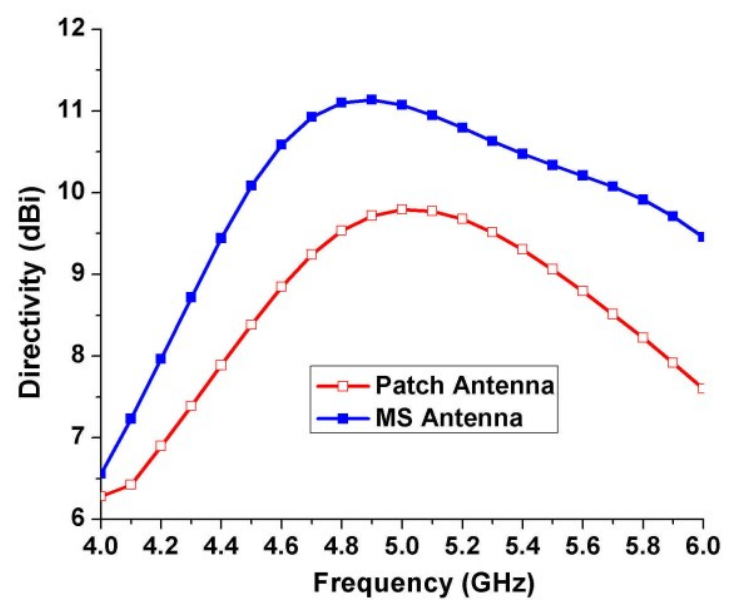

(a)

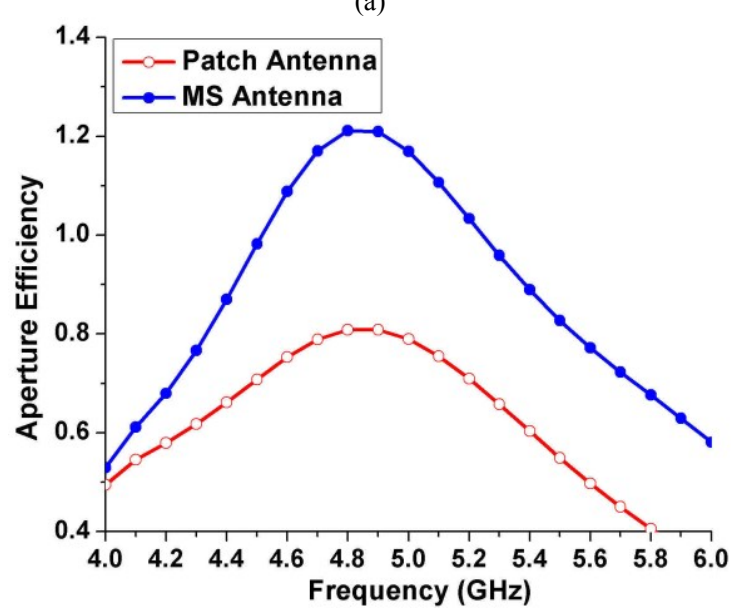

(b)

Fig. 3. Simulated (a) directivities and (b) aperture efficiencies of patch antenna and MS antenna

Although a patch antenna has been used for illustration, other antennas could produce similar results, which need more studies for verification. 


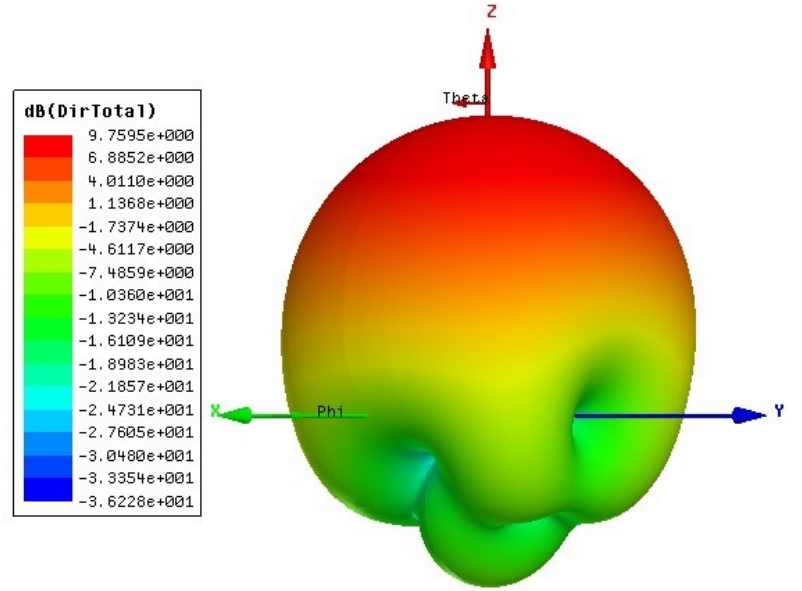

(a)

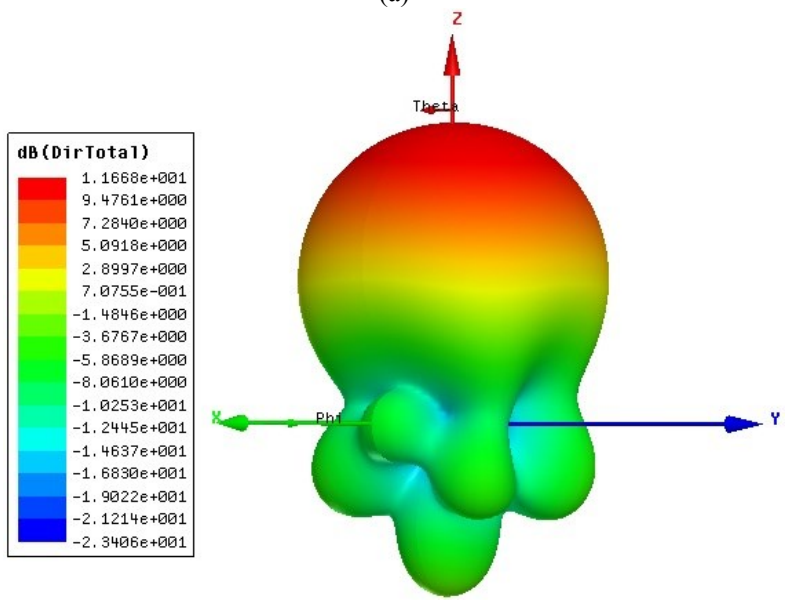

(b)
[4] X. Chen, H. Feng Ma, X. Ying Zou, W. Xiang Jiang, and $\mathrm{T}$. Jun Cui, "Three-dimensional broadband and high-directivity lens antenna made of metamaterials," Journal of Applied Physics, vol. 110, pp. -, 2011.

[5] D. T. Moore, "Gradient-index optics: a review," Applied Optics, vol. 19, pp. 1035-1038, 1980/04/01 1980.

Fig. 4. 3D radiation pattern of (a) patch antenna and (b) MS antenna

\section{CONCLUSIONS}

A method of increasing the aperture efficiency of antennas using a MS has been presented. A patch antenna has been used for illustration. Results have shown that by placing a MS at a distance atop of a patch antenna, the aperture efficiency can be increased from $78 \%$ to $117 \%$ at the operating frequency. Thus by using a MS, the effective aperture size of the patch antenna is larger than its physical aperture size.

\section{REFERENCES}

[1] C. A. Balanis, ANTENNA THEORY - ANALYSIS AND DESIGN. Hoboken, New Jersey, US: John Wiley \& Sons, Inc., 2005.

[2] J. D. Kraus and R. J. Marhefka, Antennas: For All Applications, Third Edition, 2011.

[3] C. L. Holloway, E. F. Kuester, J. A. Gordon, J. O"Hara, J. Booth, and D. R. Smith, "An Overview of the Theory and Applications of Metasurfaces: The Two-Dimensional Equivalents of Metamaterials," Antennas and Propagation Magazine, IEEE, vol. 54, pp. 10-35, 2012. 